\title{
Magnetic Phase Shift Computations for Electron Tomography
}

\author{
E. Humphrey and M. De Graef \\ Department of Materials Science and Engineering, Carnegie Mellon Univ., Pittsburgh PA 15213
}

Tomographic methods have been used for years to reconstruct three-dimensional objects, particularly in the medical community. These methods can be adapted to work with vector fields such as magnetic fields. In earlier work [1], we presented a method to reconstruct the magnetic vector potential and magnetic field of permalloy islands using a vector-field filtered backprojection method. This Lorentz microscopy based method considers the phase shift of an electron wave as it passes through the sample. When the electron wave passes through an object or magnetic field, it undergoes a phase shift, the Aharonov-Bohm phase shift, which consists of electrostatic and magnetic components, which are the projections of the electrostatic potential $V$ and the magnetic vector potential A, respectively.

More accurate iterative reconstruction methods, such as Model-Based Iterative Reconstruction (MBIR) [2] and Simultaneous Iterative Reconstruction Technique (SIRT) [3] require forward projection algorithms and numerical methods to update the simulated projections during the iteration. Forward projection models for scalar quantities, such as the electrostatic potential, are relatively well understood [4], but vector field projections are more complex.

A uniformly magnetized object can be described by a shape function $D(\mathbf{r})$ and a magnetization vector $\mathbf{M}(\mathbf{r})=M_{0} \mu(\mathbf{r}) D(\mathbf{r}) ; D(\mathbf{r})$ equals 1 inside the object and vanishes outside. We discretize the object by defining $D(\mathbf{r})$ and $\mathbf{M}(\mathbf{r})$ on a 3D cubic voxel grid. Typically, voxels are described as spacefilling cubes centered on the grid points; however, here we model the voxels as equal-volume spheres. Spheres were chosen because their projections are independent of orientation, simplifying the projection algorithm significantly. Multi-grid reconstructions can be implemented easily by varying the spherical voxel size. The spheres overlap slightly, as their radius $R \approx 1.24 a$, where $2 a$ is the cubic voxel length. In the limit of vanishing grid size, this approach is equivalent to other numerical phase shift calculation approaches. The Fourier space contribution of a single voxel to the phase shift is $\phi_{m}\left(k_{x}, k_{y}\right)=\mu_{x} S_{y}-\mu_{y} S_{x}$, where $S_{\alpha}=4 \pi^{2} R^{2} \frac{i B_{0}}{\phi_{0}} \frac{j_{1}\left(k_{\perp} R\right)}{k_{\perp}^{3}} k_{\alpha}$ are independant of projection direction and $\hat{\mu}$ are the direction cosines for the magnetization vector. The position of the voxel is taken into account by a Fourier space translation phase factor. The magnetic contribution of a single voxel to the total magnetic phase shift can thus be changed independently of the other voxels, a distinct advantage for iterative reconstruction algorithms.

The magnetic phase shift of a circular disk with radius $50 \mathrm{~nm}$, thickness $20 \mathrm{~nm}$, and an infinitely sharp vortex core is shown in Fig. 1 as a function of the spherical voxel radius $a$. While an infinitely sharp vortex core is unrealistic, it poses a a good test for our algorithm, since the phase shift should not exhibit fringing fields outside the particle, and should correctly reproduce the sharp apex of the cone. A cross section of the images is shown in Fig. 2a. The phase shift of a circular disk with the same shape and a more realistic smooth vortex core of $25 \mathrm{~nm}$ is shown in Fig. 2b. These plots show that the reconstructed phase shifts agree well with the analytical phase shifts for small voxel size.

\section{References}

[1] C. Phatak, M. Beleggia, and M. De Graef. Ultramicroscopy, 108:503, 2008.

[2] S. Venkatakrishnan, L. Drummy, M. Jackson, M. De Graef, J. Simmons, and C. Bouman. IEEE Trans. Image Proc. 2013, under review. 
[3] A. H. Andersen and A. C. Kak. Ultrasonic Imaging, 6:81, 1984.

[4] Avinash C. Kak and Malcolm Slaney. Principles of Computerized Tomographic Imaging. SIAM, 2001.

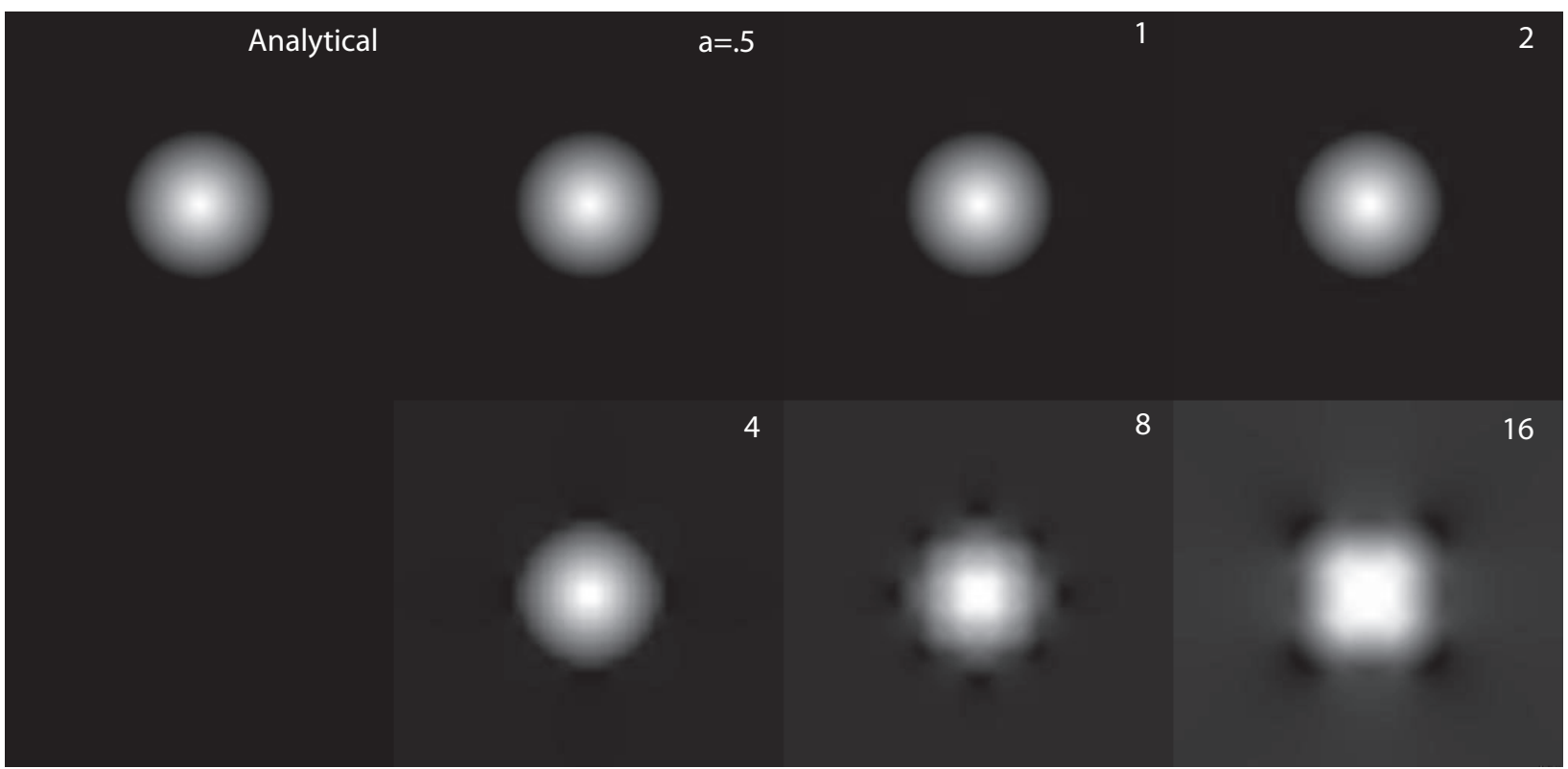

Figure 1: Analytical and multi-grid approximations of magnetic phase shift profiles of a circular disk with radius $50 \mathrm{~nm}$, thickness $20 \mathrm{~nm}$, and an infinitely sharp vortex core.

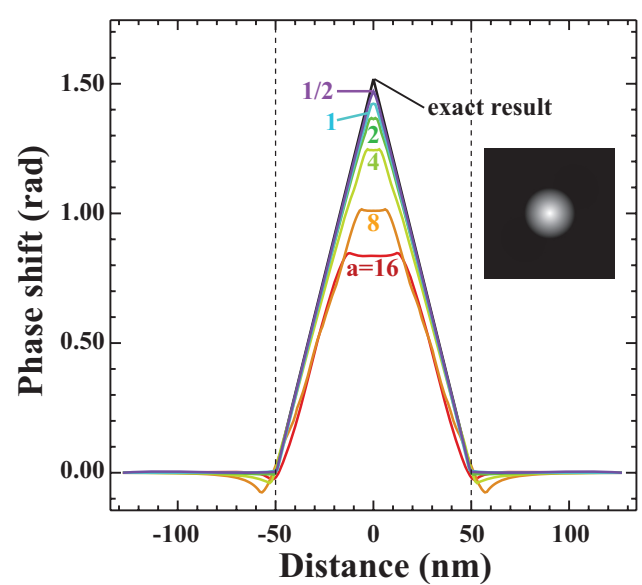

(a)

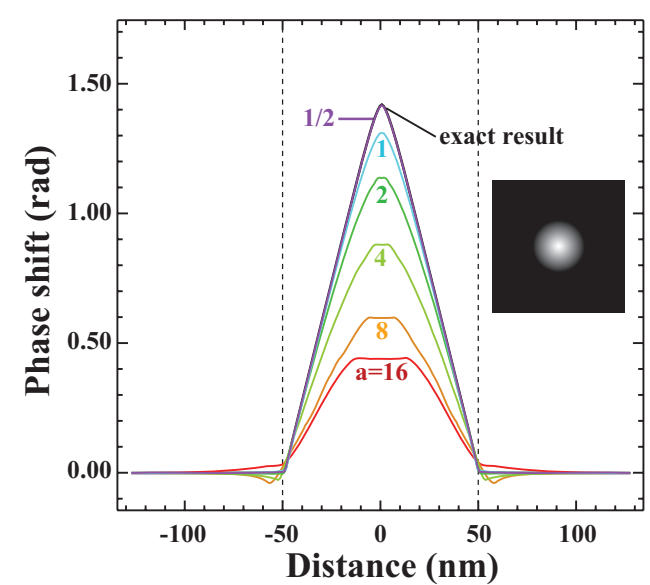

(b)

Figure 2: (a) Magnetic phase shift of the object in Fig. 1 calculated analytically (black line), and via multi-grid approximations (colored lines). The inset shows the 2D analytical phase shift. (b) Magnetic phase shift of a circular disk with radius $50 \mathrm{~nm}$, thickness $20 \mathrm{~nm}$, and vortex core $25 \mathrm{~nm}$ calculated analytically (black line), and multi-grid approximations (colored lines). 